\title{
Prolonged pituitary downregulation with gonadotropin-releasing hormone agonist improves the live-birth rate: a retrospective cohort study comparing 3 different protocols
}

\author{
Mengjia Ruan ${ }^{1}$, Luyan Fang ${ }^{1}$, Shuangqing Yang ${ }^{1}$, Zhuling Chen ${ }^{1}$, Yichen $\mathbf{W u}^{1}$, Xianqin $\mathbf{Q u}{ }^{1,2}$, \\ Junzhao Zhao ${ }^{1}$, Jing Cheng ${ }^{1 \wedge}$ \\ ${ }^{1}$ Reproductive Center, Department of Obstetrics and Gynecology, The Second Affiliated Hospital and Yuying Children's Hospital of Wenzhou \\ Medical University, Wenzhou, China; ${ }^{2}$ School of Life Sciences, Faculty of Science, University of Technology Sydney, Sydney, Australia \\ Contributions: (I) Conception and design: J Cheng, M Ruan; (II) Administrative support: J Zhao; (III) Provision of study materials or patients: J \\ Cheng, J Zhao; (IV) Collection and assembly of data: L Fang, S Yang, Z Chen, Y Wu; (V) Data analysis and interpretation: M Ruan; (VI) Manuscript \\ writing: All authors; (VII) Final approval of manuscript: All authors. \\ Correspondence to: Jing Cheng; Junzhao Zhao. Reproductive Center, Gynecology and Obstetrics Department, The Second Affiliated Hospital \\ and Yuying Children's Hospital of Wenzhou Medical University, No.306 of Hualong Qiao Road, Lucheng District, Wenzhou 325027, China. \\ Email: 01chengjing@163.com; z.joyce08@163.com.
}

Background: The long protocol has been recognized as the gold standard in controlled ovarian hyperstimulation (COH). However, the full dose of gonadotropin-releasing hormone agonist (GnRH-a) under the prolonged protocol has become increasingly popular in China. This study sought to compare pregnancy outcomes among the following 3 groups: a long protocol group, and 2 types of improved prolonged protocol groups.

Methods: A retrospective cohort study was conducted of 550 patients undergoing fresh embryo transfer (ET). Patients were treated either with the improved prolonged protocol in the follicular phase (Group 1; $\mathrm{n}=288$ ) or the mid-luteal phase (Group 2; $\mathrm{n}=143$ ), or the long protocol (Group 3; $\mathrm{n}=119$ ). The clinical and laboratory outcomes of the 3 groups were compared.

Results: The general characteristics of the women in the 3 groups were comparable. On the day on which gonadotropin (Gn) was first administered and on the day on which human chorionic gonadotropin (hCG) was administered, the luteinizing hormone (LH) levels of patients in both Groups 1 and 2 were lower than those of patients in Group 3. The number of oocytes retrieved, fertilized, and cleaved, and the number of high-quality embryos in the 3 procedures were similar. However, the number of transferred embryos, the rate of blastocyst progression, and the rate of implantation differed. The clinical pregnancy rates (CPRs) were significantly higher in the prolonged protocol groups $(62.5 \%$ and $61.5 \%)$ than the long protocol group (48.7\%). Further, statistically significant differences in the live-birth rates (LBRs) $(56.9 \%$ vs. $57.3 \%$ vs. 42.9\%) were observed. However, no differences in early abortion rates were found.

Conclusions: As a result of pituitary downregulation with GnRH-a, the prolonged groups had better CPRs and LBRs than the long protocol group. The prolonged protocol in the mid-luteal phase was equally effective as that in the early follicular phase in fresh in-vitro fertilization (IVF)/intracytoplasmic sperm injection-embryo transfer (ICSI-ET) cycles. High LH levels on the day of hCG may be a predictor of adverse clinical outcomes.

Keywords: In vitro fertilization and embryo transfer (IVF-ET); gonadotropin-releasing hormone agonist (GnRHa); pituitary downregulation; controlled ovarian hyperstimulation $(\mathrm{COH})$; live-birth rate

^ ORCID: 0000-0001-9457-159x. 
Submitted Jun 30, 2021. Accepted for publication Sep 15, 2021.

doi: 10.21037/apm-21-2335

View this article at: https://dx.doi.org/10.21037/apm-21-2335

\section{Introduction}

Infertility is one of the most concerning health problems facing all societies. It is estimated that about $70 \%$ of couples will be affected by infertility by 2025 (1). Assisted reproductive technology has become increasingly popular in treating infertility in many countries. To achieve optimal follicular and endometrial development, controlled ovarian hyperstimulation $(\mathrm{COH})$ protocols play a significant role in in-vitro fertilization and embryo transfer (IVF-ET) treatment. In clinical settings, it is essential to choose appropriate regimens according to individual conditions.

Over time, the long protocol has been recognized as the gold standard in $\mathrm{COH}$. It can be divided into two forms: the short-acting and long-acting long protocol with comparable clinical results (2). In short-acting long protocol, daily low-dose of gonadotropin-releasing hormone agonist (GnRH-a) was injected until the day of administering the human chorionic gonadotropin (hCG) injection, making it inconvenient and not acceptable widely in clinic now. The long-acting long protocol requires only a single dose of GnRH-a in the mid-luteal phase and is much more comfortable for patients. That is administration of the GnRH-a $1.875 \mathrm{mg}$ or fewer dosage commenced in the mid-luteal phase of the prior cycle (3). Under the prolonged protocol, a single dose of $3.75 \mathrm{mg}$ GnRH-a was initiated at a proper time. Women received a $3.75 \mathrm{mg}$ single dose of GnRH-a, the endogenous hormone levels can be completely inhibited within 2 weeks. Follicle-stimulating hormone (FSH) levels return to normal 3 to 4 weeks after the injection, while luteinizing hormone (LH) suppression is maintained for 8 th week (4). FSH and $\mathrm{LH}$ are indispensable for estradiol $\left(\mathrm{E}_{2}\right)$ biosynthesis and follicular oocyte development, and LH is especially indispensable during the late follicular phase (5).

Both the prolonged protocol and the long protocol are widely used; however, debate continues as to which of these approaches is better. It seems the full-dose-GnRH-a prolonged protocol has become increasingly popular in China. It has been reported that the conception rates of women who received a $3.75 \mathrm{mg} \mathrm{GnRH}$-a protocol were higher than those who received a low dose of GnRH-a daily $(2,6)$. Further, both the pregnancy rate and live-birth rate
(LBR) of women who received the prolonged regime were significantly higher than those of women who received the traditional long protocol (7). However, no information is available analysing whether these inspiring outcomes with prolonged protocol related with hormones alteration during $\mathrm{COH}$. In addition, about the time at which $\mathrm{GnRH}-\mathrm{a}$ is administered, under the prolonged regimen it can be administered at 2 different times. In the early follicular phase, the GnRH-a can be injected between the 2nd and 5 th days of the menstrual cycle, while in the mid-luteal phase, it can be administered in the middle of the previous luteal cycle.

This retrospective study examined the effects of both the traditional long protocol and the 2 above-mentioned improved prolonged protocols. The primary objective of this study was to compare pregnancy outcomes, including the CPR, early abortion rate, and LBR among the 3 groups. The secondary objective was to assess the correlations between hormones during $\mathrm{COH}$ with pregnancy outcomes.

We present the following article in accordance with the STROBE reporting checklist (available at https://dx.doi. org/10.21037/apm-21-2335).

\section{Methods}

\section{Patients}

In total, 550 women, who underwent IVF or received intracytoplasmic sperm injections (ICSIs) from June 2017 to December 2019 at the Reproductive Center of The Second Affiliated Hospital of Wenzhou Medical University, were included in this study. Patients were treated either with the improved prolonged protocol in the follicular phase (Group 1; $\mathrm{n}=288$ ) or the mid-luteal phase (Group 2; n=143), or the long protocol (Group 3; $\mathrm{n}=119$ ). To be eligible to participate in this study, patients had to meet the following inclusion criteria: (I) be a female aged $\leq 40$ years; and (II) have a baseline FSH $<10 \mathrm{IU} / \mathrm{L}$. Patients were excluded from this study if they met any of the following exclusion criteria: (I) had a body mass index (BMI) $>30 \mathrm{~kg} / \mathrm{m}^{2}$; and/or (II) had endometriosis. All procedures performed in this study involving human participants were in accordance with the Declaration of Helsinki (as revised in 2013). The study was 

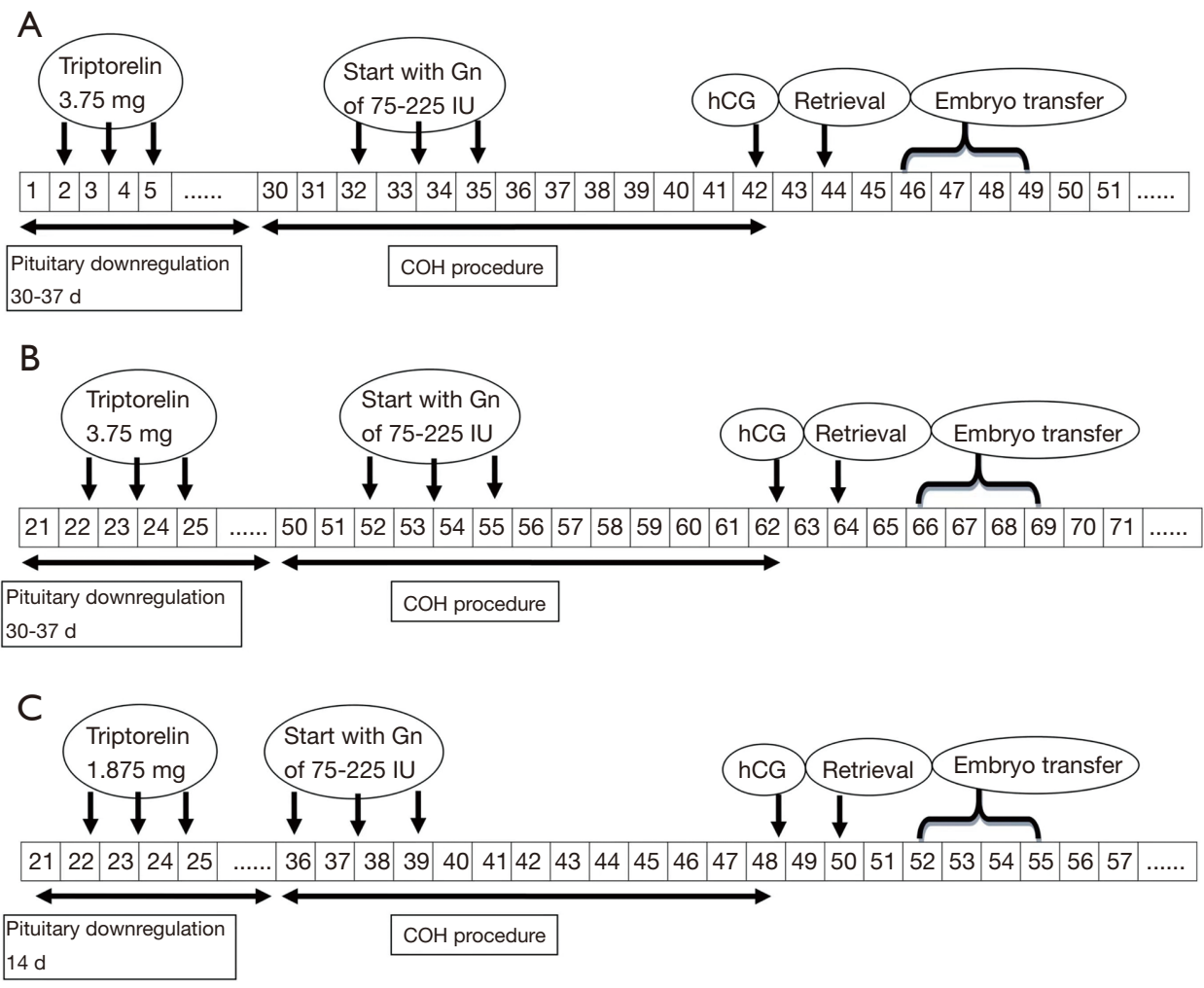

Figure 1 Flow chart of the 3 stimulation protocols. (A) GnRH agonist prolonged regimen in Group 1: patients were given an injection of $3.75 \mathrm{mg}$ of GnRH-a during the 2 nd to 5 th day of menstruation cycle. Ovarian stimulation with $75-225$ IU Gn would start 3037 days later along with confirmation of pituitary downregulation. COH procedure was adjusted according to patients' ovarian response. A 5,000/10,000 IU injection of hCG was performed when at least one dominant follicle reached diameters of 18 mm or two follicles reached $17 \mathrm{~mm}$ in diameter, and then oocytes were retrieved 36-38 h later. After that, embryo transfer was performed 2-5 days later. Luteal phase support was sustained from the day of oocyte retrieval. (B) GnRH agonist prolonged regimen in Group 2: patients were given an injection of $3.75 \mathrm{mg}$ of GnRH-a during mid-luteal phase of last menstrual cycle, the rest procedures were similar to those of Group 1. (C) GnRH agonist long regimen in Group 3: patients were given an injection of half dose (1.875 $\mathrm{mg})$ of GnRH-a in a single intramuscular injection in the mid-luteal phase (days 22-25) of the menstrual cycle. Down-regulation was confirmed after 14 days following the same criteria described in the prolonged protocol and was followed by $\mathrm{Gn}$ in stimulation. The rest procedures were similar to those of Group 1.

approved by ethics board of The Second Affiliated Hospital and Yuying Children's Hospital of Wenzhou Medical University (No.: 2021-K-70-02) and informed consent was taken from all the patients.

\section{Improved prolonged protocol}

In Group 1, patients were given an injection of $3.75 \mathrm{mg}$ of GnRH-a (Triptorelin; Ferring, Kiel, Germany) during the 2 nd to 5 th days of their menstruation cycle (see Figure 1A). In Group 2, pituitary downregulation was achieved in the mid-luteal phase via a single full dose of $3.75 \mathrm{mg}$ of triptorelin (see Figure 1B);30-37 days later, pituitary downregulation was achieved if they met the following criteria: $\mathrm{E}_{2} \leq 30 \mathrm{pg} / \mathrm{mL}, \mathrm{LH} \leq 5 \mathrm{IU} / \mathrm{L}$, an ovarian follicle diameter $\leq 5 \mathrm{~mm}$, and an endometrial thickness $\leq 5 \mathrm{~mm}$. A daily dose of 75-225 IU of gonadotropin (Gn) was initiated for ovarian stimulation. The dose was adjusted in accordance with hormonal level changes and the growth of the follicles. To trigger the final maturation of the follicles, a 5,000/10,000 IU injection of hCG (Livzon, Guangdong, China) was administered when at least 1 dominant follicle 
reached a diameter of $18 \mathrm{~mm}$ or 2 follicles reached a diameter of $17 \mathrm{~mm}$.

\section{Long protocol}

In Group 3, patients received the GnRH-a long protocol. Specifically, patients received a $1 / 2$ dose $(1.875 \mathrm{mg})$ of GnRH-a (Triptorelin; Ferring, Kiel, Germany) via a single intramuscular injection in the mid-luteal phase (days 22-25) of the menstrual cycle. A preceding treatment was used for pituitary downregulation. Downregulation was confirmed after 14 days using the same criteria described in the prolonged protocol and was followed by Gn stimulation (see Figure 1C).

\section{IVF/ICSI procedure}

Oocytes were retrieved 36-38 hours after trigger of hCG. Then oocytes were inseminated through IVF or ICSI 4-6 hours after oocyte collection. The preceding criteria (Veeck, 1986) (8) was taken to assess the grade the embryos. It was agreed that embryos would be scored in four categories according to the cell number, fragmentation, symmetry and so on. Embryos of Veeck grades 1 or 2 were graded as high quality. Blastocyst scoring included the density, expansion stage of the blastocyst cavity, the cell number of the inner cell mass, and the cohesion and regularity of the trophectoderm (9). The blastocyst progression rate was calculated as the ratio of the number of blastocysts over the number of embryos for cryopreservation.

With the assistance of a trans-abdominal ultrasound, ET was performed with an early blastocyst or blastocyst. Luteal phase support was sustained via the oral administration of $20 \mathrm{mg}$ of dydrogesterone (Duphaston, Mylan Medical, France) per day, in addition to $90 \mathrm{mg}$ of progesterone vaginal gel prolonged release each day (Crinone; Merck Serono, Hertfordshire, United Kingdom), starting from the day following oocyte retrieval.

\section{Pregnancy outcome}

Clinical pregnancy was defined as a transvaginal ultrasound detection of an intrauterine gestational sac on days $27-28$ after ET. The implantation rate was calculated as the ratio of the number of gestational sac(s) over the number of transferred fresh embryos. Early abortion was defined as a clinical pregnancy that failed to reach the 12th gestational week. Live-birth was defined as the birth of a live child after 28 weeks of gestation.

\section{Statistical methods}

Data were analyzed using SPSS (version 23.0, USA). The statistical differences among the continuous variables were evaluated using analyses of variance or Kruskal-Wallis $\mathrm{H}$ tests as required. The categorical variables were analyzed using Chi-square tests or Fisher's exacts test as required. A $\mathrm{P}<0.05$ was considered statistically significant.

\section{Results}

The general characteristics of the women in the 3 groups were comparable (see Table 1). There were also no differences in their basic $\mathrm{LH}, \mathrm{FSH}$, and $\mathrm{E}_{2}$ levels on day 3. However, on the Gn commencing day, the LH levels of patients in both Groups 1 and 2 were lower than those of patients in Group 3 ( $0.40 \pm 0.19$ vs. $0.32 \pm 0.20$ vs. $1.20 \pm 0.74 \mathrm{IU} / \mathrm{L} ; \mathrm{P}<0.001)$, which suggested a stronger suppression on pituitary and ovaries. Additionally, Group 3 had the lowest progesterone $\left(\mathrm{P}_{4}\right)$ levels, which showed a significant difference. Patients' antral follicular count (AFC) numbers on the commencing day were equivalent among the groups.

On the day of hCG, patients' in Groups 1 and 2 had lower LH levels than those in Group $3(0.68 \pm 0.44 v s$. $0.71 \pm 0.49$ vs. $0.89 \pm 0.55$ IU/L; $\mathrm{P}<0.001$; see Table 1 ). However, in terms of the $\mathrm{E}_{2}$ and $\mathrm{P}_{4}$ levels, there were no differences among the 3 groups. Patients' endometrial thickness measurements on the day of hCG were also equivalent. The days of stimulation did not differ among the groups; however, the total dose of Gn was statistically different among 3 groups $(\mathrm{P}=0.005)$. More doses were needed to in Group 3 than in Groups 1 and 2.

A comparison of the laboratory outcomes showed that the number of oocytes retrieved, fertilized, and cleaved, and the number of high-quality embryos in the 3 procedures were similar (see Table 2). Based on the patients' requirements, there was a difference in the number of transferred embryos $(1.83 \pm 0.37$ vs. $1.38 \pm 0.49$ vs. $1.81 \pm 0.49 ; \mathrm{P}<0.001)$. In addition, in Group 2 , the rate of blastocyst progression and implantation rate were statistically higher than in Groups 1 and 3.

As expected, the CPRs were significantly higher in the prolonged protocol groups $(62.5 \%$ and $61.5 \%)$ than the long protocol group (48.7\%) (see Table 2). Further, a statistically significant difference in LBR $(56.9 \%$ vs. $57.3 \%$ 
Table 1 Patients' basic characteristics and the stimulation variables for the 3 groups

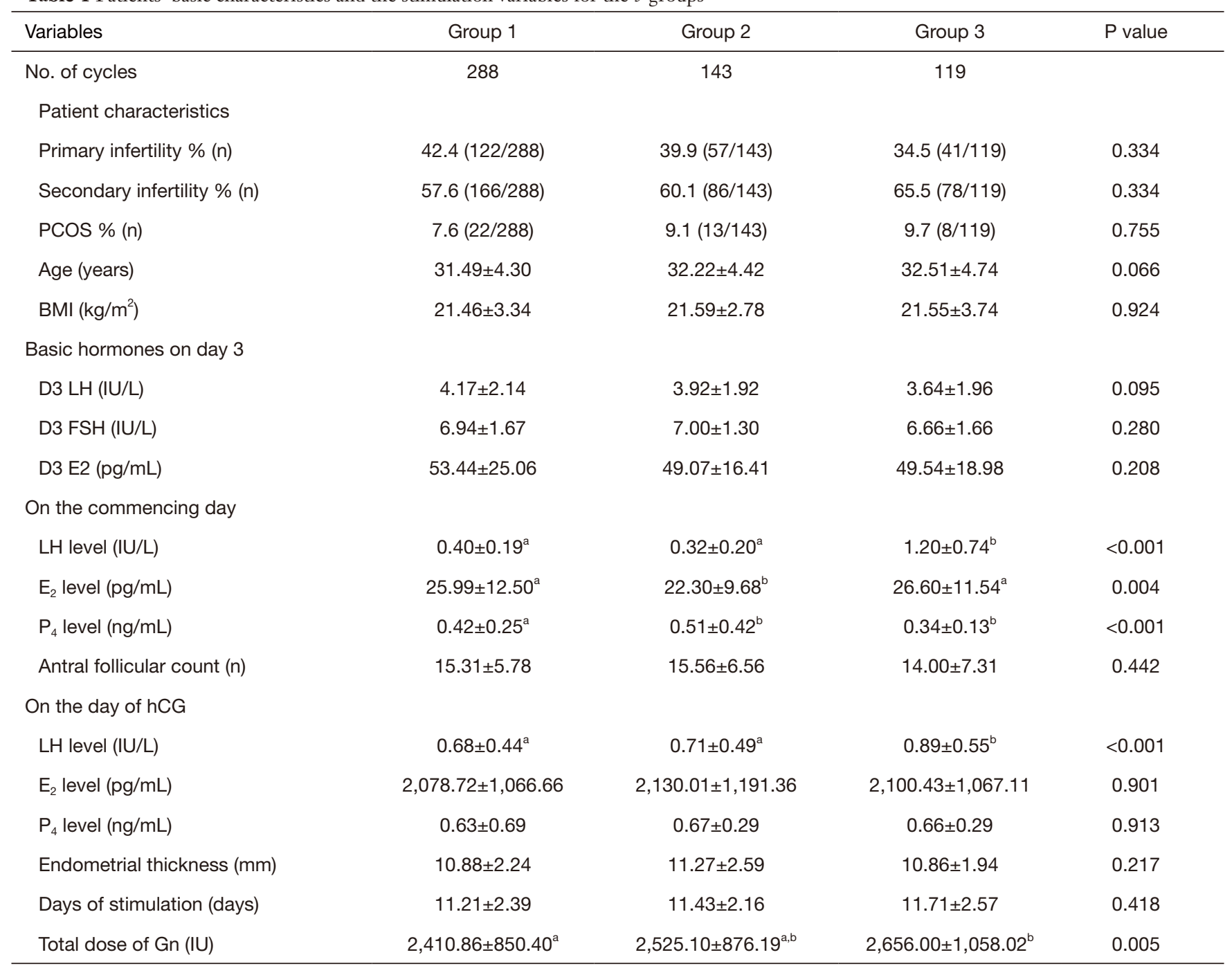

${ }^{a, b}$, groups with a different letter differ significantly $(\mathrm{P}<0.05)$. PCOS, polycystic ovary syndrome; LH, luteinizing hormone; FSH, follicle-stimulating hormone; hCG, human chorionic gonadotropin.

vs. $42.9 \%$ ) was also found between Groups 1 and 2 and Group 3. However, there were no differences in the early abortion rates among the groups. The results of further comparisons among the 3 groups are shown in Figure 2.

\section{Discussion}

The process of downregulation plays a crucial role in the clinical outcomes of IVF. Most previous studies have focused on comparing 2 protocols with GnRH-a. Ren et al. found that a prolonged protocol with a full dose of $\mathrm{GnRH}-\mathrm{a}$ starting in the early follicular phase resulted in a higher LBR than that of a long protocol with a $1 / 3$ dose $(1.25 \mathrm{mg})$ of GnRH-a in the mid-luteal phase (7). This study was the first to compare 3 different protocols to further explore the effects of different doses and starting times of GnRH-a. In addition, we explored rate of blastocyst progression in this study, and found it was higher in prolonged protocol with a full dose of GnRH-a during mid-luteal phase than that from long protocol, which is differ from that previous report (7).

LH concentrations could affect the quality of oocytes and pregnancy outcomes. Humaidan put forward the theory of a "window," which suggested that LH levels should neither be too high or too low during a course of $\mathrm{COH}$ (10). It has been reported that higher basic LH levels in polycystic ovary syndrome (PCOS) women are closely related to early 
Table 2 Laboratory and clinical outcomes of the 3 groups

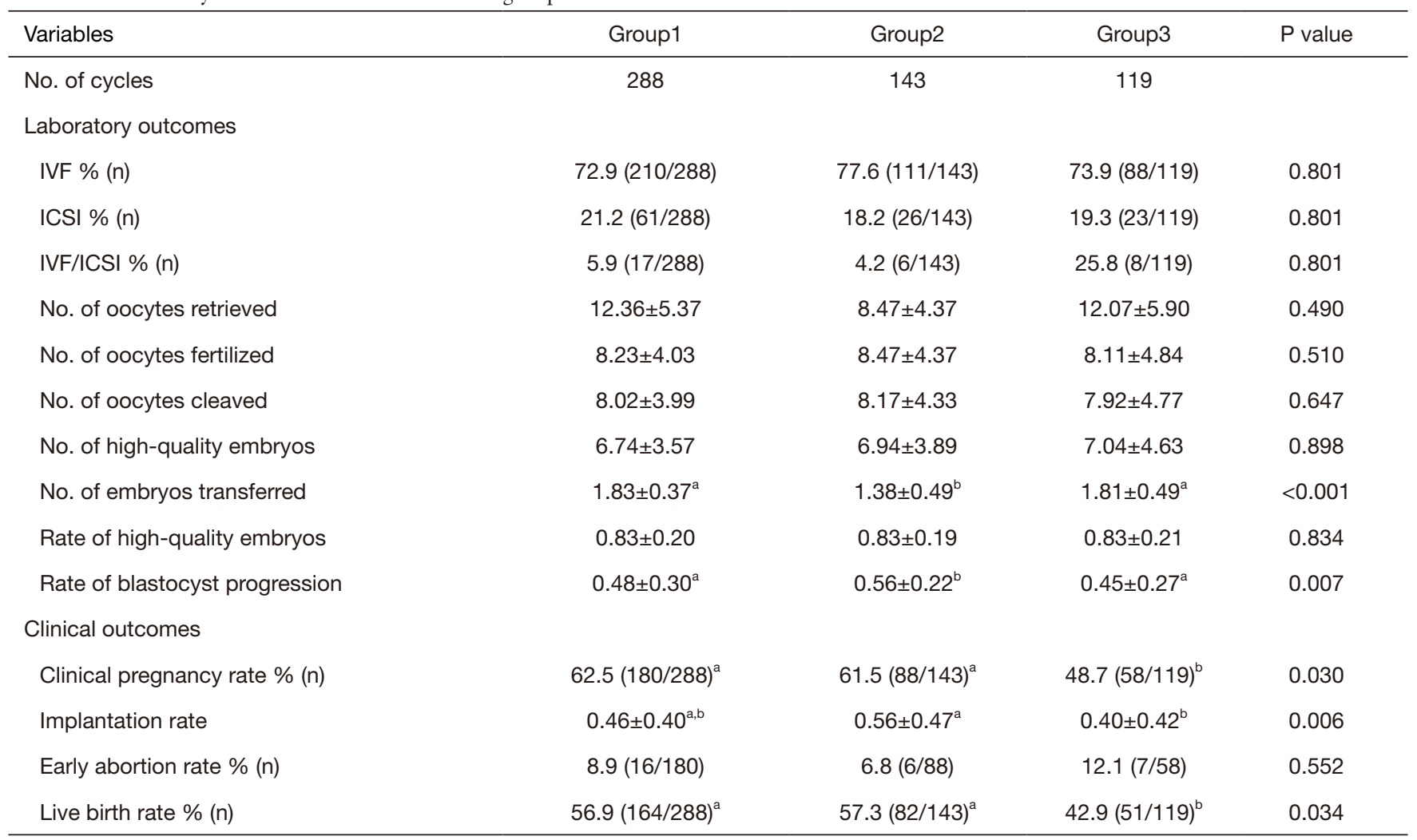

${ }^{a, b}$, groups with a different letter differ significantly $(P<0.05)$.
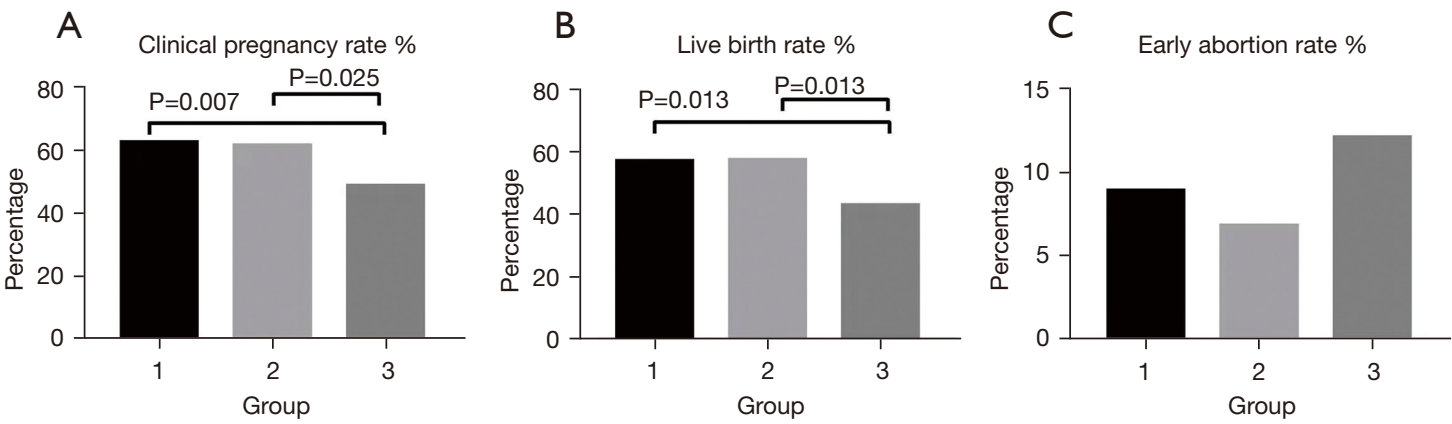

Figure 2 Pregnancy outcomes for the 3 different groups. According to the further comparisons among the 3 groups, it showed the clinical pregnancy rate were significantly higher in the prolonged protocol groups $(62.5 \%$ and $61.5 \%)$ than the long protocol group $(48.7 \%)(\mathrm{P}=0.007$, $\mathrm{P}=0.025)$. Further, a statistically significant difference in live birth rate (56.9\% vs. $57.3 \%$ vs. $42.9 \%)$ was also found between Groups 1 and 2 and Group $3(\mathrm{P}=0.013, \mathrm{P}=0.013)$. However, there were no differences in the early abortion rates among the groups.

pregnancy loss (11). In addition, early pregnancy loss has been shown to occur in women with normal menstrual cycles whose LH is $>10$ IU/L during the early follicular phase (12). Conversely, Westergaard et al. distinguished between "low" and "high" LH levels with a cutoff of
$0.5 \mathrm{mIU} / \mathrm{mL}$ after 8 days of $\mathrm{COH}$, and found a 5 -fold increase in early pregnancy loss in the "low" LH group, which was directly related to a decrease of LBR (13). In a recent study, patients undergoing the long protocol were divided into the following 4 groups based on their LH levels 
on Gn stimulation day 8: (I) <0.5 IU/L; (II) 0.51-1.0 IU/L; (III) $1.01-1.5 \mathrm{IU} / \mathrm{L}$; and (IV) $>1.51 \mathrm{IU} / \mathrm{L}$. The results suggested that fertilization and clinical pregnancies were superior in the middle 2 groups (10). Collectively, LH levels affect pregnancy outcomes directly or indirectly. However, the optimal LH range remains controversial.

In the present study, patients' LH levels on the day on which $\mathrm{Gn}$ administration was commenced in the improved prolonged protocol groups were lower than those of patients in the long protocol group, which suggested a stronger suppression on pituitary and ovaries. On the day of hCG, patients' LH levels in the improved prolonged protocol groups remained lower than those in the long protocol group $(\mathrm{P}<0.001)$. Our results are consistent with those of Ren's, who compared a prolonged protocol in the early follicular phase to a long protocol in the mid-luteal phase (7). Like Ying et al. (14), we found that were no significant differences in the hormone levels of patients in the 2 improved prolonged protocol groups.

Several studies have found that a $1 / 2(15)$ or $1 / 3$ dose (16) of depot triptorelin was sufficient to prevent $\mathrm{LH}$ surges and enhance the ovarian response. Compared to patients downregulated with a $1 / 2$ dose of depot triptorelin in the long protocol group, patients in the prolonged protocol group required more $\mathrm{Gn}$ and yielded fewer oocytes (17). However, the number of high-quality embryos was unaffected (7). Our data simultaneously showed that there were no differences in the number of oocytes retrieved, fertilized, and cleaved, and the number of high-quality embryos in the 3 procedures.

The thickness of the endometrium has been recognized as a prognostic factor to evaluate endometrial receptivity and embryo transplantation (18). Previous studies suggested through regulation of specific enzymes and cytokines, GnRH-a treatment may exert effects on the endometrium (19). GnRH-a appears to regulate expression of endometrial interleukin 1 system, $\alpha v \beta 3$ vitronectin, and vascular endothelial growth factor (20,21). Furthermore, endometrial receptivity markers such as homeobox A10, myeloid ecotropic viral integration site 1 and leukemia inhibitory factor in endometrium were all showed statistically higher in the depot GnRH-a protocol than in other protocols (17). Most studies demonstrated significantly higher pregnancy rate along with the endometrial thickness grew, irrespective of the number or quality of the embryos $(22,23)$. Additionally, an endometrial thickness of more than $7 \mathrm{~mm}$ was optimal for clinical pregnancy outcomes (24).
Regrettably, results from this study showed the endometrial thickness on the hCG day was comparable among 3 different groups.

Besides endometrial thickness, reactivity of ovary is another important factor affects CPR and LBR as well as safety of patients. A recently review combined and analysed seven studies, including proportions of normal ovarian responders, polycystic ovary syndrome (PCOS), and poor responders. Despite increased risk of ovarian hyperstimulation syndrome (OHSS), the long-acting GnRH agonist follicular protocol was still beneficial in improving live birth rate than GnRH antagonist protocol (25).

To ensure the optimization of a single blastocyst, we preferred to transfer 2 D3 embryos or 1 D5 blastocyst. A larger percentage ofD5 blastocysts was transferred in Group 2, which accounted for the lower number of transferred embryos in Group 2 than the other 2 groups $(\mathrm{P}<0.001)$. It may be that the higher rate of blastocyst progression and the higher implantation rate in Group 2 is related to the low number of transferred embryos.

In Ren et al.'s study (7), a statistically significantly higher implantation rate $(50.27 \%$ vs. $39.69 \%)$, CPR (64.02\% vs. $56.87 \%)$, and LBR per fresh transfer cycle $(55.56 \%$ vs. $45.73 \%$ ) was observed in the prolonged protocol group compared to long protocol group. As expected, the results of the present study reflect those of previous studies (7). In addition, a further comparison of the 2 different types of prolonged protocols revealed no differences in pregnancy outcomes. These results are similar to those of Ying et al.'s (14). In relation to hormone levels and pregnancy outcomes, high LH levels on the day of hCG may be a predictor of adverse clinical outcomes.

Together, though so many protocols in IVF-ET cycle, downregulated with GnRH-a has always been a first choice. Not only increased CPR and LBR as in this study, increased the number of euploid embryos (6) and increased clinical pregnancy and liver birth rate for those with thin endometrium make prolonged protocol more and more popular. It might be associated with exposure time to GnRH-a (26), although the precise mechanism remains unclear.

The study was limited by its retrospective nature and its specified patient cohort, which consisted of young, normogonadotropic women. In addition, the present study only focused on the effects of fresh ET and did not examine frozen ET. This study is a retrospective analysis, which is likely to cause some deviations in the results. It needs to be further confirmed by multi-center clinical trials. 


\section{Conclusions}

As a result of pituitary downregulation with $\mathrm{GnRH}-\mathrm{a}$, the prolonged groups had better CPRs and LBRs than the long protocol group. The prolonged protocol in the mid-luteal phase was equally effective as that in the early follicular phase in fresh IVF/ICSI-ET cycles. High LH levels on the day of hCG may be a predictor of adverse clinical outcomes. These results have value for IVF/ICSI treatments.

\section{Acknowledgments}

Funding: (I) Health Commission of Zhejiang Province (2018KY521); (II) Zhejiang Provincial Clinical Medicine Key Level Discipline Open Fund of Wenzhou Medical University (LKFJ040); (III) Doctoral Research Initiation Fund of The Second Affiliated Hospital of Wenzhou Medical University.

\section{Footnote}

Reporting Checklist: The authors have completed the STROBE reporting checklist. Available at https://dx.doi. org/10.21037/apm-21-2335

Data Sharing Statement: Available at https://dx.doi. org/10.21037/apm-21-2335

Conflicts of Interest: All authors have completed the ICMJE uniform disclosure form (available at https://dx.doi. org/10.21037/apm-21-2335). All authors reported that the study was partly supported by funding from Health Commission of Zhejiang Province (2018KY521), Zhejiang Provincial Clinical Medicine Key Level Discipline Open Fund of Wenzhou Medical University ( LKFJ040), Doctoral Research Initiation Fund of the Second Affiliated Hospital of Wenzhou Medical University and with the participation of Medical Ethics Committee of the Second Affiliated Hospital and Yuying Children's Hospital of Wenzhou Medical University that gave the approval (2021-k-70-72). The authors have no other conflicts of interest to declare.

Ethical Statement: The authors are accountable for all aspects of the work in ensuring that questions related to the accuracy or integrity of any part of the work are appropriately investigated and resolved. All procedures performed in this study involving human participants were in accordance with the Declaration of Helsinki (as revised in 2013). The study was approved by ethics board of The Second Affiliated Hospital and Yuying Children's Hospital of Wenzhou Medical University (No.: 2021-K-70-02) and informed consent was taken from all the patients.

Open Access Statement: This is an Open Access article distributed in accordance with the Creative Commons Attribution-NonCommercial-NoDerivs 4.0 International License (CC BY-NC-ND 4.0), which permits the noncommercial replication and distribution of the article with the strict proviso that no changes or edits are made and the original work is properly cited (including links to both the formal publication through the relevant DOI and the license). See: https://creativecommons.org/licenses/by-nc-nd/4.0/.

\section{References}

1. Zahmatkeshan $M$, Naghdi $M$, Farjam $M$, et al. ART Registries-Characteristics and experiences: A comparative study. J Family Med Prim Care 2019;8:449-54.

2. Song J, Duan C, Cai W, et al. Comparison of GnRH-a Prolonged Protocol and Short GnRH-a Long Protocol in Patients with Thin Endometrium for Assisted Reproduction: A Retrospective Cohort Study. Drug Des Devel Ther 2020;14:3673-82.

3. Depalo R, Jayakrishan K, Garruti G, et al. GnRH agonist versus $\mathrm{GnRH}$ antagonist in in vitro fertilization and embryo transfer (IVF/ET). Reprod Biol Endocrinol 2012;10:26.

4. Broekmans FJ, Bernardus RE, Berkhout G, et al. Pituitary and ovarian suppression after early follicular and mid-luteal administration of a LHRH agonist in a depot formulation: decapeptyl CR. Gynecol Endocrinol 1992;6:153-61.

5. Chen C, Yan Q, Liu K, et al. Endometrial Receptivity Markers in Mice Stimulated With Raloxifene Versus Clomiphene Citrate and Natural Cycles. Reprod Sci 2016;23:748-55.

6. Li G, Wu Y, Niu W, et al. Analysis of the Number of Euploid Embryos in Preimplantation Genetic Testing Cycles With Early-Follicular Phase Long-Acting Gonadotropin-Releasing Hormone Agonist Long Protocol. Front Endocrinol (Lausanne) 2020;11:424.

7. Ren J, Sha A, Han D, et al. Does prolonged pituitary down-regulation with gonadotropin-releasing hormone agonist improve the live-birth rate in in vitro fertilization treatment? Fertil Steril 2014;102:75-81.

8. Rosenwaks Z, Veeck LL, Liu HC. Pregnancy following transfer of in vitro fertilized donated oocytes. Fertil Steril 
1986;45:417-20.

9. Alpha Scientists in Reproductive Medicine and ESHRE Special Interest Group of Embryology. The Istanbul consensus workshop on embryo assessment: proceedings of an expert meeting. Hum Reprod 2011;26:1270-83.

10. Humaidan P, Bungum L, Bungum M, et al. Ovarian response and pregnancy outcome related to mid-follicular LH levels in women undergoing assisted reproduction with $\mathrm{GnRH}$ agonist down-regulation and recombinant FSH stimulation. Hum Reprod 2002;17:2016-21.

11. Johnson P, Pearce JM. Recurrent spontaneous abortion and polycystic ovarian disease: comparison of two regimens to induce ovulation. BMJ 1990;300:154-6.

12. Regan L, Owen EJ, Jacobs HS. Hypersecretion of luteinising hormone, infertility, and miscarriage. Lancet 1990;336:1141-4.

13. Westergaard LG, Laursen SB, Andersen CY. Increased risk of early pregnancy loss by profound suppression of luteinizing hormone during ovarian stimulation in normogonadotrophic women undergoing assisted reproduction. Hum Reprod 2000;15:1003-8.

14. Ying Y, Yang T, Zhang H, et al. Prolonged pituitary down-regulation with full-dose of gonadotropinreleasing hormone agonist in different menstrual cycles: a retrospective cohort study. PeerJ 2019;7:e6837.

15. Dal Prato L, Borini A, Coticchio G, et al. Half-dose depot triptorelin in pituitary suppression for multiple ovarian stimulation in assisted reproduction technology: a randomized study. Hum Reprod 2004;19:2200-5.

16. Li Y, Yang D, Zhang Q. Clinical outcome of one-thirddose depot triptorelin is the same as half-dose depot triptorelin in the long protocol of controlled ovarian stimulation. J Hum Reprod Sci 2012;5:14-9.

17. Xu B, Geerts D, Hu S, et al. The depot GnRH agonist protocol improves the live birth rate per fresh embryo transfer cycle, but not the cumulative live birth rate in normal responders: a randomized controlled trial and molecular mechanism study. Hum Reprod 2020;35:1306-18.

18. Nakamura K, Oosawa M, Kondou I, et al. Menotropin stimulation after prolonged gonadotropin releasing

Cite this article as: Ruan M, Fang L, Yang S, Chen Z, Wu Y, Qu X, Zhao J, Cheng J. Prolonged pituitary downregulation with gonadotropin-releasing hormone agonist improves the live-birth rate: a retrospective cohort study comparing 3 different protocols. Ann Palliat Med 2021;10(9):9984-9992. doi: 10.21037/apm-21-2335 hormone agonist pretreatment for in vitro fertilization in patients with endometriosis. J Assist Reprod Genet 1992;9:113-7.

19. Khan KN, Kitajima M, Hiraki K, et al. Changes in tissue inflammation, angiogenesis and apoptosis in endometriosis, adenomyosis and uterine myoma after $\mathrm{GnRH}$ agonist therapy. Hum Reprod 2010;25:642-53.

20. Meresman GF, Bilotas MA, Lombardi E, et al. Effect of $\mathrm{GnRH}$ analogues on apoptosis and release of interleukin1 beta and vascular endothelial growth factor in endometrial cell cultures from patients with endometriosis. Hum Reprod 2003;18:1767-71.

21. Raga F, Casañ EM, Bonilla-Musoles F. Gonadotropinreleasing hormone $(\mathrm{GnRH})-\mathrm{I}$ regulation of interleukin (IL)-1b and IL-1 receptor antagonist expression in cultured human endometrial stromal cells. J Obstet Gynaecol Res 2008;34:464-72.

22. Al-Ghamdi A, Coskun S, Al-Hassan S, et al. The correlation between endometrial thickness and outcome of in vitro fertilization and embryo transfer (IVF-ET) outcome. Reprod Biol Endocrinol 2008;6:37.

23. Chen SL, Wu FR, Luo C, et al. Combined analysis of endometrial thickness and pattern in predicting outcome of in vitro fertilization and embryo transfer: a retrospective cohort study. Reprod Biol Endocrinol 2010;8:30.

24. Wang Y, Zhu Y, Sun Y, et al. Ideal embryo transfer position and endometrial thickness in IVF embryo transfer treatment. Int J Gynaecol Obstet 2018;143:282-8.

25. Yang R, Guan Y, Perrot V, et al. Comparison of the LongActing GnRH Agonist Follicular Protocol with the GnRH Antagonist Protocol in Women Undergoing In Vitro Fertilization: A Systematic Review and Meta-analysis. Adv Ther 2021;38:2027-37.

26. Song J, Wu W, Jiang L, et al. Effects of Different Exposure Days to Gonadotropin-Releasing Hormone Agonist (GnRH-a) on Live Birth Rates in the Depot GnRH-a Protocol: A Retrospective Analysis of 7007 Cycles. Med Sci Monit 2021;27:e929854.

(English Language Editor: L. Huleatt) 